\title{
COLOURING AND COVERING NOWHERE DENSE GRAPHS*
}

\author{
MARTIN GROHE ${ }^{\dagger}$, STEPHAN KREUTZER $^{\ddagger}$, ROMAN RABINOVICH $^{\S}$, \\ SEBASTIAN SIEBERTZ $ף$ AND KONSTANTINOS STAVROPOULOS"
}

\begin{abstract}
In [12] it was shown that nowhere dense classes of graphs admit sparse neighbourhood covers of small degree. We show that a monotone graph class admits sparse neighbourhood covers if and only if it is nowhere dense. The existence of such covers for nowhere dense classes is established through bounds on so-called weak colouring numbers. The core results of this paper are various lower and upper bounds on the weak colouring numbers and other, closely related generalised colouring numbers. We prove tight bounds for these numbers on graphs of bounded treewidth. We clarify and tighten the relation between the density of shallow minors and the various generalised colouring numbers. These upper bounds are complemented by new, stronger exponential lower bounds on the weak and strong colouring numbers, and by super-polynomial lower bounds on the weak colouring numbers on classes of polynomial expansion. Finally, we show that computing weak $r$-colouring numbers is NP-complete for all $r \geq 3$.
\end{abstract}

1. Introduction. Nowhere dense classes of graphs have been introduced by Nešetřil and Ossona de Mendez $[18,20]$ as a general model of uniformly sparse graph classes. They include and generalise many other natural sparse graph classes, among them all classes of bounded degree, classes of bounded genus, classes defined by excluded (topological) minors, and classes of bounded expansion. It has been demonstrated in several papers, e.g., $[2,8,12,16,18]$ that nowhere dense graph classes have nice algorithmic properties; many problems that are hard in general can be solved (more) efficiently on nowhere dense graph classes. In fact, nowhere dense classes are a natural limit for the efficient solvability of a wide class of problems [7, 12, 15].

In [12], it was shown that nowhere dense classes of graphs admit sparse neighbourhood covers. Neighbourhood covers play an important role in the study of distributed network algorithms and other application areas (see for example [21]). The neighbourhood covers developed in [12] combine low radius and low degree making them interesting for the applications outlined above. In this paper, we prove a (partial) converse to the result of [12]: we show that monotone graph classes (that is, classes closed under taking subgraphs) are nowhere dense if and only if they admit sparse neighbourhood covers. A similar characterisation result was proved for classes of bounded expansion [19].

Nowhere denseness has turned out to be a very robust property of graph classes with various seemingly unrelated characterisations (see [11, 18]), among them characterisations through so-called generalised colouring numbers. These are particularly relevant in the algorithmic context, because the existence of sparse neighbourhood covers for nowhere dense

${ }^{*}$ A conference version of this paper was published at the 41st International Workshop on Graph-Theoretic Concepts in Computer Science, WG 2015. The jounal version contains new additional results. Stephan Kreutzer and Roman Rabinovich's research has been supported by the European Research Council (ERC) under the European Union's Horizon 2020 research and innovation programme (ERC Consolidator Grant DISTRUCT, grant agreement No 648527). The work of Sebastian Siebertz is supported by the National Science Centre of Poland via POLONEZ grant agreement UMO-2015/19/P/ST6/03998, which has received funding from the European Union's Horizon 2020 research and innovation programme (Marie Skłodowska-Curie grant agreement No. 665778).

${ }^{\dagger}$ RWTH Aachen University, grohe@informatik.rwth-aachen.de

$\ddagger$ Technische Universität Berlin, stephan.kreutzer@tu-berlin.de

$\S$ Technische Universität Berlin, roman.rabinovich@tu-berlin.de

IUniversity of Warsaw, siebertz@mimuw.edu.pl

\|Universität Hamburg, konstantinos.stavropoulos@uni-hamburg.de 
classes is established through such colouring numbers-the weak r-colouring numbers, to be precise - and the value of these numbers is directly related to the degree of the neighbourhood covers. Besides the weak $r$-colouring numbers $\operatorname{wcol}_{r}(G)$ of graphs $G$ we study the $r$-colouring numbers $\operatorname{col}_{r}(G)$ and the $r$-admissibility numbers $\operatorname{adm}_{r}(G)$. The two families of colouring numbers where introduced by Kierstead and Yang in [14], and the admissibility numbers go back to Kierstead and Trotter in [13] and were generalised by Dvořák in [6]. All these numbers generalise the degeneracy, a.k.a. colouring number, which is defined to be the minimum $d$ such that there is a linear order of the vertices of $G$ in which every vertex has at most $d$ smaller neighbours. The name "colouring number" comes from the fact that graphs of degeneracy $d$ have a proper $d+1$ colouring which can be computed efficiently by a simple greedy algorithm. For the generalised $r$-colouring numbers, instead of smaller neighbours of a vertex we count smaller vertices reachable by certain paths of length $r$; the numbers differ by the kind of paths of length $r$ considered. We observe that with growing $r$ the colouring numbers converge to the treewidth of the graph.

The core results of this paper are various upper and lower bounds for these families of colouring numbers. In particular, we prove tight bounds for $\mathrm{wcol}_{r}(G)$ for graphs $G$ of bounded treewidth. We clarify and tighten the relation between the density of shallow minors and the various generalised colouring numbers. These upper bounds are complemented by new, stronger exponential lower bounds on the strong and weak colouring numbers. The lower bounds can already be achieved on graph classes of bounded degree. We furthermore show that there exist classes of polynomial expansion on which the weak colouring numbers grow super-polynomially in $r$. This result answers negatively a question of Joret and Wood, whether graph classes of polynomial expansion have polynomial weak colouring numbers. Finally, we show that computing weak $r$-colouring numbers is NP-complete for all $r \geq 3$.

After giving some graph theoretic background in Section 2, we prove our various bounds on the generalised colouring numbers in Sections 3-5. Section 6 is devoted to sparse neighbourhood covers, and the NP-completeness result for the weak colouring numbers is proved in Section 7.

2. Generalised Colouring Numbers. Our notation from graph theory is standard, we refer the reader to [3] for background. All graphs in this paper are finite and simple, i.e. they do not have self-loops or multiple edges. A class of graphs is monotone if it is closed under subgraphs. The radius $\operatorname{rad}(G)$ of $G$ is $\min _{u \in V(G)} \max _{v \in V(G)} \operatorname{dist}^{G}(u, v)$. By $N_{r}^{G}(v)$ we denote the $r$-neighbourhood of $v$ in $G$, i.e. the set of vertices of distance at most $r$ from $v$ in $G$.

We represent a linear order on $V(G)$ as an injective function $L: V(G) \rightarrow \mathbb{N}$ and write $\Pi(G)$ for the set of all linear orders on $V(G)$.

Vertex $u$ is weakly $r$-reachable from $v$ with respect to the order $L$, if there is a path $P$ of length $0 \leq \ell \leq r$ from $v$ to $u$ such that $L(u) \leq L(w)$ for all $w \in V(P)$. Let WReach $[G, L, v]$ be the set of vertices that are weakly $r$-reachable from $v$ with respect to $L$. If furthermore, all inner vertices $w$ of $P$ satisfy $L(v)<L(w)$, then $u$ is called strongly r-reachable from $v$. Let $\operatorname{SReach}_{r}[G, L, v]$ be the set of vertices that are strongly $r$-reachable from $v$ with respect to $L$.

The $r$-admissibility $\operatorname{adm}_{r}[G, L, v]$ of $v$ with respect to $L$ is the maximum size $k$ of a family $\left\{P_{1}, \ldots, P_{k}\right\}$ of paths of length at most $r$ in $G$ that start in $v$, end at a vertex $w$ with $L(w) \leq L(v)$ and satisfy $V\left(P_{i}\right) \cap V\left(P_{j}\right)=\{v\}$ for $1 \leq i \neq j \leq k$. As we can always let the paths end in the first vertex smaller than $v$, we can assume that the internal vertices of the paths are larger than $v$. Note that $\operatorname{adm}_{r}[G, L, v]$ is an integer, whereas $\operatorname{WReach}_{r}[G, L, v]$ and $\operatorname{SReach}_{r}[G, L, v]$ are sets of vertices. 
The weak $r$-colouring number $\operatorname{wcol}_{r}(G)$, the $r$-colouring number $\operatorname{col}_{r}(G)$, and the $r$ admissibility $\operatorname{adm}_{r}(G)$ are defined as

$$
\begin{aligned}
\operatorname{wcol}_{r}(G) & =\min _{L \in \Pi(G)} \max _{v \in V(G)}\left|\operatorname{WReach}_{r}[G, L, v]\right|, \\
\operatorname{col}_{r}(G) & =\min _{L \in \Pi(G)} \max _{v \in V(G)}\left|\operatorname{SReach}_{r}[G, L, v]\right|, \\
\operatorname{adm}_{r}(G) & =\min _{L \in \Pi(G)} \max _{v \in V(G)} \operatorname{adm}_{r}[G, L, v] .
\end{aligned}
$$

It follows from the definitions that, for all $r \in \mathbb{N}, \operatorname{adm}_{r}(G) \leq \operatorname{col}_{r}(G) \leq \operatorname{wcol}_{r}(G)$. Also, $\operatorname{adm}_{1}(G) \leq \operatorname{adm}_{2}(G) \leq \ldots \leq \operatorname{adm}_{n}(G), \operatorname{wcol}_{1}(G) \leq \operatorname{wcol}_{2}(G) \leq \ldots \leq \operatorname{wcol}_{n}(G)=\operatorname{td}(G)$ (where $\operatorname{td}(G)$ is the treedepth of $G$, see e.g. [18]) and $\operatorname{col}_{1}(G) \leq \operatorname{col}_{2}(G) \leq \ldots \leq \operatorname{col}_{n}(G)=$ $\operatorname{tw}(G)+1$ (where $\operatorname{tw}(G)$ is the treewidth of $G$ ).

To see that $\operatorname{col}_{n}(G)=\operatorname{tw}(G)+1$, note that treewidth can be characterised by elimination orders. An elimination order of a graph $G$ is a linear order $L$ on $V(G)$ with which we associate a sequence of graphs $G_{i}$. Let $V(G)=\{1, \ldots, n\}$ and $L(i)<L(j)$ for $i<j$, then $G_{0}=G$ and for $0<i \leq n, V\left(G_{i}\right)=V\left(G_{i-1}\right) \backslash\{i\}$ and

$$
E\left(G_{i}\right)=\left(E\left(G_{i-1}\right) \backslash\{\{i, j\}: j \leq n\}\right) \cup\left\{\{\ell, j\}: \ell \neq j,\{\ell, i\},\{i, j\} \in E\left(G_{i-1}\right)\right\},
$$

i.e. we eliminate vertex $i$ and make a clique out of the neighbours of $i$ in $G_{i-1}$. The width of the elimination order is the maximum size of a clique over all $G_{i}$ minus one. The elimination width of $G$ is the minimum width over all possible widths of elimination orders of $G$. It is well known that the treewidth of $G$ is equal to its elimination width. Let $L^{\prime}$ be the reverse to $L$. An easy induction shows that the neighbours of a vertex $i$ in $G_{i-1}$ are exactly those of $\operatorname{SReach}_{n}\left[G, L^{\prime}, i\right] \backslash\{i\}$. It follows that $\operatorname{col}_{n}(G)=\operatorname{tw}(G)+1$.

Furthermore, it was shown that the generalised colouring numbers are strongly related, i.e. $\operatorname{col}_{r}(G) \leq\left(\operatorname{adm}_{r}(G)-1\right) \cdot\left(\operatorname{adm}_{r}(G)-2\right)^{r-1}+1$ and $\operatorname{wcol}_{r}(G) \leq \operatorname{adm}_{r}(G)^{r}$ (see for example [6], but note that in that work, paths of length 0 are not considered for the $r$-admissibility).

3. Admissibility and Density of Shallow Minors. A graph $H$ with vertex set $V(H)=\left\{v_{1}, \ldots, v_{n}\right\}$ is a minor of a graph $G$, written $H \preceq G$, if there are pairwise vertex disjoint connected subgraphs $H_{1}, \ldots, H_{n}$ of $G$ such that whenever $v_{i} v_{j} \in E(H)$, then there are $u_{i} \in V\left(H_{i}\right)$ and $u_{j} \in\left(H_{j}\right)$ with $u_{i} u_{j} \in E(G)$. We call $\left(H_{1}, \ldots, H_{n}\right)$ a minor model of $H$ in $G$. For $r \in \mathbb{N}$, the graph $H$ is a depth-r minor of $G$, denoted $H \preceq_{r} G$, if there is a minor model $\left(H_{1}, \ldots, H_{n}\right)$ of $H$ in $G$ such that each $H_{i}$ has radius at most $r$.

For $r \in \mathbb{N}$, an $r$-subdivision of a graph $H$ is obtained from $H$ by replacing edges by pairwise internally disjoint paths of length at most $r+1$. If a graph $G$ contains a $2 r$-subdivision of $H$ as a subgraph, then $H$ is a topological depth-r minor of $G$, written $H \preceq_{r}^{t} G$. Recall that $H$ is a topological minor of $G$ (we write $H \preceq^{t} G$ ) if some subdivision of $H$ is a subgraph of $G$, that is, if $H \preceq_{r}^{t} G$ for some $r \in \mathbb{N}$.

The edge density of a graph $G$ is $\varepsilon(G)=|E(G)| /|V(G)|$. Note that the average degree of $G$ is $2 \varepsilon(G)$. A graph is $k$-degenerate if every subgraph has a vertex of degree at most $k$. The maximum of the edge densities of all $H \preceq_{r} G$ is known as the greatest reduced average density $\nabla_{r}(G)$ of $G$ with rank $r$. Similarly, the maximum of the edge densities of all $H \preceq_{r}^{t} G$ is known as the topological greatest reduced average density $\widetilde{\nabla}_{r}(G)$ of $G$ with $\operatorname{rank} r$. We will also 
refer to the functions $r \mapsto \nabla_{r}(G)$ and $r \mapsto \widetilde{\nabla}_{r}(G)$ as expansion and topological expansion of $G$, respectively. As proved in [5], these measures satisfy $\widetilde{\nabla}_{r}(G) \leq \nabla_{r}(G) \leq 4\left(4 \widetilde{\nabla}_{r}(G)\right)^{(r+1)^{2}}$.

A class $\mathcal{C}$ of graphs is nowhere dense if for all $\varepsilon>0$ and all $r \in \mathbb{N}$ there is an $n_{0} \in \mathbb{N}$ such that all $n$-vertex graphs $G \in \mathcal{C}$ with at least $n_{0}$ vertices satisfy $\nabla_{r}(G) \leq n^{\varepsilon} . \mathcal{C}$ is said to have bounded expansion if for every $r$ there is a $c(r)$ such that $\nabla_{r}(G) \leq c(r)$ for all $G \in \mathcal{C}$. It is easy to see that all classes of bounded expansion are nowhere dense; the converse does not hold. We say that $\mathcal{C}$ has polynomial expansion if there is a polynomial $p(x)$ such that $\nabla_{r}(G) \leq p(r)$ for all $r \in \mathbb{N}$ and $G \in \mathcal{C}$.

The following theorem implies improvements of previous results from Kierstead and Yang [14] and Zhu [25] to the exponent of their upper bounds for colouring numbers and the weak colouring numbers.

Theorem 3.1. Let $G$ be a graph and let $r \geq 1$. Then $\operatorname{adm}_{r}(G) \leq 6 r\left(\widetilde{\nabla}_{r-1}(G)\right)^{3}$.

Every class $\mathcal{C}$ that excludes a topological minor has $\widetilde{\nabla}_{r}(G)$ bounded by a universal constant for every $G \in \mathcal{C}$. This includes familiar classes such as classes of bounded degree, bounded genus, and bounded treewidth. We obtain the following corollary.

COROLlary 3.2. Let $\mathcal{C}$ be a graph class that excludes some fixed graph as a topological minor. Then for all $G \in \mathcal{C}$ we have $\operatorname{adm}_{r}(G) \in \mathcal{O}(r)$ and $\operatorname{wcol}_{r}(G) \in \mathcal{O}\left(\left(c_{\mathcal{C}} \cdot r\right)^{r}\right)$, where $c_{\mathcal{C}}$ is a constant depending only on the class $\mathcal{C}$.

For the proof of Theorem 3.1 we need a lemma which is a variation of a result of Dvořák [6]. For a set $S \subseteq V(G)$ and $v \in S$, let $b_{r}(S, v)$ be the maximum number $k$ of paths $P_{1}, \ldots, P_{k}$ of length at most $r$ from $v$ to $S$ with internal vertices in $V(G) \backslash S$ and with $V\left(P_{i}\right) \cap V\left(P_{j}\right)=\{v\}$ for $1 \leq i \neq j \leq k$.

Lemma 3.3 ([6]). For all graphs $G$ and $r \in \mathbb{N}$, there exists a set $S \subseteq V(G)$ such that $b_{r}(S, v) \geq \operatorname{adm}_{r}(G)$ for all $v \in S$.

Proof. Assume that all subsets $S \subseteq V(G)$ contain a vertex $v$ such that $b_{r}(S, v)<\operatorname{adm}_{r}(G)$. We construct an order $L\left(v_{1}\right)<L\left(v_{2}\right)<\ldots<L\left(v_{n}\right)$ of $V(G)$ as follows. If $v_{i+1}, \ldots, v_{n}$ have already been ordered, choose $v_{i}$ such that if $S_{i}=\left\{v_{1}, \ldots, v_{i}\right\}$, then $b_{r}\left(S_{i}, v_{i}\right)$ is minimal. Clearly, the $r$-admissibility of the resulting order is one of the values $b_{r}\left(S_{i}, v_{i}\right)$ occurring in its construction. This implies $\operatorname{adm}_{r}(G)<\operatorname{adm}_{r}(G)$, a contradiction.

Proof of Theorem 3.1. Let $G$ be a graph with $\widetilde{\nabla}_{r-1}(G) \leq c$, and let $\ell:=6 r c^{3}+1$. Suppose for contradiction that $\operatorname{adm}_{r}(G)>\ell$. By Lemma 3.3, there exists a set $S$ such that $b_{r}(S, v)>\ell$ for all $v \in S$. For $v \in S$, let $\mathcal{P}_{v}$ be a set of paths from $v$ to $S$ witnessing this, and let $s:=|S|$.

Choose a maximal set $\mathcal{P}$ of pairwise internally vertex-disjoint paths of length at most $2 r-1$ connecting pairs of vertices from $S$ whose internal vertices belong to $V(G) \backslash S$ such that each pair of vertices is connected by at most one path. Let $H$ be the graph with vertex set $S$ and edges between all vertices $v, w \in S$ connected by a path in $\mathcal{P}$. Then $H \preceq_{r-1}^{t} G$ and hence $|\mathcal{P}|=|E(H)| \leq s \cdot c$. Let $M$ be the set of all internal vertices of the paths in $\mathcal{P}$, and let $m:=|M|$. Then $m \leq s \cdot c \cdot(2 r-2)$.

Note that we not only have $H \preceq_{r-1}^{t} G$, but also $H^{\prime} \preceq_{r-1}^{t} G$ for all $H^{\prime} \subseteq H$. Thus for all $H^{\prime} \subseteq H$ we have $\varepsilon\left(H^{\prime}\right) \leq c$, and therefore $H^{\prime}$ has a vertex of degree at most $2 c$. In other words, $H$ is $2 c$-degenerate. This implies that $H$ is $(2 c+1)$-colourable and hence contains an independent set $R$ of size at least $\lceil s /(2 c+1)\rceil$. 
For $v \in S$, let $\mathcal{Q}_{v}$ be the set of initial segments of paths in $\mathcal{P}_{v}$ from $v$ to a vertex in $(M \cup S) \backslash\{v\}$ with all internal vertices in $V(G) \backslash(M \cup S)$. Observe that for $u, v \in R$ the paths in $\mathcal{Q}_{v}$ and $\mathcal{Q}_{u}$ are internally disjoint, because if $Q \in \mathcal{Q}_{u}$ and $Q^{\prime} \in \mathcal{Q}_{v}$ had an internal vertex in common, then $Q \cup Q^{\prime}$ would contain a path of length at most $2 r-2$ that is internally disjoint from all paths in $\mathcal{P}$, contradicting maximality of $\mathcal{P}$.

Let $G^{\prime}$ be the union of all paths in $\mathcal{P}$ and all paths in $\mathcal{Q}_{v}$ for $v \in R$, and let $H^{\prime}$ be obtained from $G^{\prime}$ by contracting all paths in $\bigcup_{v \in R} \mathcal{Q}_{v}$ to single edges. Then $H^{\prime} \preceq_{r-1}^{t} G$.

We have $\left|V\left(H^{\prime}\right)\right| \leq s+m \leq s+s \cdot c \cdot(2 r-2) \leq s \cdot c \cdot(2 r-1)$ and at least $\left|E\left(H^{\prime}\right)\right| \geq$ $\lceil s /(2 c+1)\rceil \cdot \ell$ edges. Thus $\varepsilon\left(H^{\prime}\right) \geq \ell / 6 r c^{2}>c$. A contradiction.

4. The Weak Colouring Numbers of Graphs of Bounded Treewidth. A tree decomposition of a graph $G$ is a pair $(T, X)$, where $T$ is a tree, $X=\left(X_{t}: t \in V(T)\right)$, is a family of subsets of $\mathrm{V}(\mathrm{G})$ (called bags) such that

(i) $\bigcup_{t \in V(T)} X_{t}=V(G)$,

(ii) for every edge $\{u, v\}$ of $G$ there exists $t \in V(T)$ with $u, v \in X_{t}$ and

(iii) if $r, s, t \in V(T)$ and $s$ lies on the unique path of $T$ between $r$ and $t$, then $X_{r} \cap X_{t} \subseteq X_{s}$.

A graph has treewidth at most $k$ if it admits a tree decomposition $(T, X)$ such that $\left|X_{t}\right| \leq k+1$ for each $t \in V(T)$ and we write $\operatorname{tw}(G)$ for the treewidth of $G$. We assume familiarity with the basic theory of tree decompositions as in [3].

It is well known that a graph of treewidth $k$ has a tree decomposition $(T, X)$ of width $k$ such that for every $\{s, t\} \in E(T)$ we have $\left|X_{s} \backslash X_{t}\right| \leq 1$. We call such decompositions smooth. The following separation property of tree decompositions is well known.

Lemma 4.1. If $r, s, t \in V(T), u \in X_{r}$ and $v \in X_{t}$ and $s$ is on the path of $T$ between $r$ and $t$, then every path from $u$ to $v$ in $G$ uses a vertex contained in $X_{s}$.

For a tree decomposition $(T, X)$ of $G$ and a node $s \in V(T)$ we define a partial order $L^{T, s}$ on $V(T)$ demanding that $L^{T, s}(t) \leq L^{T, s}(u)$ if $t$ lies on the path from $s$ to $u$ (i.e. $L^{T, s}$ is the standard tree order where $s$ is minimum).

Theorem 4.2. Let $\operatorname{tw}(G) \leq k$. Then $\operatorname{wcol}_{r}(G) \leq\left(\begin{array}{c}r+k \\ k\end{array}\right)$.

Proof. Let $(T, X)$ be a smooth tree decomposition of $G$ of width at most $k$. Since if $G^{\prime}$ is a subgraph of $G$, then $\operatorname{wcol}_{r}\left(G^{\prime}\right) \leq \operatorname{wcol}_{r}(G)$, w.l.o.g. we may assume that $G$ is edge maximal of treewidth $k$, i.e. each bag induces a clique in $G$. We choose an arbitrary root $s$ of $T$ and let $L^{\prime}$ be some linear extension of $L^{T, s}$. For every $v \in V(G)$, let $t_{v}$ be the unique node of $T$ such that $L^{\prime}\left(t_{v}\right)=\min \left\{L^{\prime}(t) \mid v \in X_{t}\right\}$ and define a linear ordering $L:=L_{G}^{T, s}$ of $V(G)$ such that:

(i) $L^{\prime}\left(t_{v}\right)<L^{\prime}\left(t_{u}\right) \Rightarrow L(v)<L(u)$, and

(ii) if $L^{\prime}\left(t_{v}\right)=L^{\prime}\left(t_{u}\right)$ (which is possible in the root bag $X_{s}$ ), break ties arbitrarily.

Fix some $v \in V(G)$ and let $w \in \operatorname{WReach}_{r}[G, L, v]$. By Lemma 4.1 and the definition of $L$, it is immediate that $t_{w}$ lies on the path from $t_{v}$ to $s$ in $T$. Let $u \in X_{t_{v}}$ be such that $L(u) \leq L\left(u^{\prime}\right)$ for all $u^{\prime} \in X_{t_{v}}$. If $t_{v}=s$, then $\mid$ WReach $_{r}[G, L, v] \mid \leq k+1$ and we are done. Otherwise, as the decomposition is smooth, $L^{\prime}\left(t_{u}\right)<L^{\prime}\left(t_{v}\right)$. We define two subgraphs $G_{1}$ and $G_{2}$ of $G$ as follows. The graph $G_{1}$ is induced by the vertices from the bags between $s$ and $t_{u}$, i.e. by the set $\bigcup\left\{X_{t} \in V(T): L^{T, s}(t) \leq L^{T, s}\left(t_{u}\right)\right\}$. The graph $G_{2}$ is induced by $\bigcup\left\{X_{t} \in V(T): L^{T, s}\left(t_{u}\right) \leq L^{T, s}(t)<L^{T, s}\left(t_{v}\right)\right\} \backslash V\left(G_{1}\right)$. 
Let $L_{i}$ be the restriction of $L$ to $V\left(G_{i}\right)$, for $i=1,2$, respectively. We claim that if $w \in \operatorname{WReach}_{r}[G, L, v]$, then $w \in \operatorname{WReach}_{r-1}\left[G_{1}, L_{1}, u\right] \cup \operatorname{WReach}_{r}\left[G_{2}, L_{2}, v\right]$. To see this, let $P=\left(v=v_{1}, \ldots, v_{\ell}=w\right)$ be a shortest path between $v$ and $w$ of length $\ell \leq r$ such that $L(w)$ is minimum among all vertices of $V(P)$.

We claim that $L\left(v_{1}\right)>\ldots>L\left(v_{\ell}\right)$ (and call $P$ a decreasing path). This implies in particular that all $t_{v_{i}}$ lie on the path from $t_{v}$ to $s$ and that $L^{T, s}\left(t_{v_{1}}\right) \geq \ldots \geq L^{T, s}\left(t_{v_{\ell}}\right)$ (non-equality may only hold in the last step, if we take a step in the root bag).

Assume that the claim does not hold and let $i$ be the first position with $L\left(v_{i}\right)<L\left(v_{i+1}\right)$. It suffices to show that we can find a subsequence (which is also a path in $G$ ) $Q=v_{i}, v_{j}, \ldots, v$ of $P$ with $j>i+1$. By definition of $t_{v_{i+1}}=: t, X_{t}$ contains $v_{i}$. (Indeed, there is an edge between $v_{i}$ and $v_{i+1}$, which must be contained in some bag, but $v_{i+1}$ appears first in $X_{t}$ counting from the root and each bag induces a clique in $G$ ). Let $t^{\prime}$ be the parent node of $t . X_{t^{\prime}}$ also contains $v_{i}$, as the decomposition is smooth and $v_{i+1}$ is the unique vertex that joins $X_{t}$. But by Lemma 4.1, $X_{t^{\prime}}$ is a separator that separates $v_{i+1}$ from all vertices smaller than $v_{i+1}$. We hence must visit another vertex $v_{j}$ from $X_{t^{\prime}}$ in order to finally reach $v$. We can therefore shorten the path as claimed.

If $L(w) \leq L(u)$, then $P$ goes through $X_{t_{u}}$ by Lemma 4.1. Let $u^{\prime}$ be the first vertex of $P$ that lies in $X_{t_{u}}$. We show that there is a shortest path from $v$ to $u^{\prime}$ that uses $u$ as the second vertex. By assumption, $v \neq u$. If $\left\{v, u^{\prime}\right\} \in E(G)$, then $\left\{v, u^{\prime}\right\}$ must be contained in some bag $X_{t^{\prime}}$. By definition of $t_{v}, t^{\prime}=t_{v}$, as $t_{v}$ is the first node of $T$ on the path from $s$ to $t_{v}$ containing $v$. By definition of $t_{u}$ and because $(T, X)$ is smooth, $u$ is the only vertex from $t_{v}$ that appears in $t_{u}$. Thus $u^{\prime}=u$, so the shortest path from $v$ to $u^{\prime}$ uses $u$. If the distance between $v$ and $u^{\prime}$ is at least 2, a shortest path can be chosen as $v, u, u^{\prime}$. Indeed $u \in X_{t_{u}} \cap X_{t_{v}}$ and every bag induces a clique by assumption.

It follows that if $L(w) \leq L(u)$ and $w \in \operatorname{WReach}_{r}[G, L, v]$, then there is a shortest path from $v$ to $w$ that uses $u$ as the second vertex. Thus $w \in \operatorname{WReach}_{r-1}\left[G_{1}, L_{1}, u\right]$, as $P$ is decreasing.

If $L(w)>L(u)$, then $P$ never visits vertices of $G_{1}$. If $P$ lies completely in $G_{2}$, we have $w \in$ WReach $_{r}\left[G_{2}, L_{2}, v\right]$. If $P$ leaves $G_{2}$, it visits vertices of $G$ that are contained only in bags strictly below $t_{v}$. However, this is impossible, as $P$ is decreasing.

Therefore we have

$$
\left|\operatorname{WReach}_{r}[G, L, v]\right| \leq\left|\operatorname{WReach}_{r-1}\left[G_{1}, L_{1}, u\right]\right|+\left|\operatorname{WReach}_{r}\left[G_{2}, L_{2}, v\right]\right| .
$$

Note that the treewidth of $G_{2}$ is at most $k-1$, as we removed $u$ from every bag. More precisely, the tree decomposition $\left(T^{2}, X^{2}\right)$ of $G_{2}$ of width at most $k-1$ is the restriction of $(T, X)$ to $G_{2}$, i.e. we take tree nodes $t$ contained between $t_{u}$ and $t_{v}$ (including $t_{v}$ and not including $\left.t_{u}\right)$ and define $X_{t}^{2}=X_{t} \cap V\left(G_{2}\right)$.

Now, recall the definition of $L_{G}^{T, s}$ as in the beginning of the proof and let $w(r, k)$ be the maximum $\mid$ WReach $_{r}\left[H, L_{H}^{T, s}, v\right] \mid$, ranging over all graphs $H$ with $\operatorname{tw}(H) \leq k$, linear orders $L_{H}^{T, s}$ obtained by an $s \in V(T)$, and vertices $v \in V(H)$. By $(\star)$, we then have $\mid$ WReach $_{r}[G, L, v] \mid \leq w(r, k-1)+w(r-1, k)$. Since $G, L$ and $v$ where arbitrary, it follows that

$$
w(k, r) \leq w(k, r-1)+w(k-1, r) .
$$

Recall that wcol $_{1}(G)$ equals the degeneracy of $G$ plus one and note that every graph of treewidth $\leq k$ is $k$-degenerate, hence $w(k, 1) \leq k+1$. Furthermore, it is easy to observe that 
for a tree $T$, we have $\operatorname{wcol}_{r}(T) \leq r+1$ : any linear extension of a tree-order with respect to some root will do. Hence $w(1, r) \leq r+1$. Since $\left(\begin{array}{c}r+k \\ k\end{array}\right)=\left(\begin{array}{c}r+k-1 \\ k\end{array}\right)+\left(\begin{array}{c}r+k-1 \\ k-1\end{array}\right)$, we conclude by induction that $w(r, k) \leq\left(\begin{array}{c}r+k \\ k\end{array}\right)$.

The proof of Theorem 4.2 gives rise to a construction of a class of graphs that matches the upper bound proven there. We construct a graph of treewidth $k$ and weak $r$-colouring number $\left(\begin{array}{c}k+r \\ k\end{array}\right)$ whose tree decomposition $(T, X)$ has a highly branching host tree $T$. This enforces a path in the tree from the root to a leaf that realises the recursion from the proof of Theorem 4.2 .

THEOREM 4.3. For every $k \geq 1, r \geq 1$, there is a family of graphs $G_{r}^{k}$ with $\operatorname{tw}\left(G_{r}^{k}\right)=k$, such that $\operatorname{wcol}_{r}\left(G_{r}^{k}\right)=\left(\begin{array}{c}r+k \\ k\end{array}\right)$. In fact, for all $r^{\prime} \leq r, \operatorname{wcol}_{r^{\prime}}\left(G_{r}^{k}\right)=\left(\begin{array}{c}r^{\prime}+k \\ k\end{array}\right)$.

Proof. Fix $r, k$ and let $c=\left(\begin{array}{c}r+k \\ k\end{array}\right)$. We define graphs $G\left(k^{\prime}, r^{\prime}\right)$ for all $r^{\prime} \leq r, k^{\prime} \leq k$ and corresponding tree decompositions $\mathcal{T}\left(k^{\prime}, r^{\prime}\right)=\left(T\left(k^{\prime}, r^{\prime}\right), X\left(k^{\prime}, r^{\prime}\right)\right)$ of $G\left(k^{\prime}, r^{\prime}\right)$ of width $k^{\prime}$ with a distinguished root $s\left(T\left(k^{\prime}, r^{\prime}\right)\right)$ by induction on $k^{\prime}$ and $r^{\prime}$. We will show that $\operatorname{wcol}_{r^{\prime}}\left(G\left(k^{\prime}, r^{\prime}\right)\right) \geq$ $\left(\begin{array}{c}r^{\prime}+k^{\prime} \\ k^{\prime}\end{array}\right)$. We guarantee several invariants for all values of $k^{\prime}$ and $r^{\prime}$ which will give us control over a sufficiently large part of any order that witnesses $\operatorname{wcol}_{r^{\prime}}\left(G\left(k^{\prime}, r^{\prime}\right)\right) \geq\left(\begin{array}{c}r^{\prime}+k^{\prime} \\ k^{\prime}\end{array}\right)$.

(i) There is a bijection $f: V\left(T\left(k^{\prime}, r^{\prime}\right)\right) \rightarrow V\left(G\left(k^{\prime}, r^{\prime}\right)\right)$ such that $f\left(s\left(T\left(k^{\prime}, r^{\prime}\right)\right)\right)$ is the unique vertex contained in $X_{s\left(T\left(k^{\prime}, r^{\prime}\right)\right)}$ and if $t$ is a child of $t^{\prime}$ in $T\left(k^{\prime}, r^{\prime}\right)$, then $f(t)$ is the unique vertex of $X_{t} \backslash X_{t^{\prime}}$. Hence any order defined on $V(T)$ directly translates to an order of $V(G)$ and vice versa.

(ii) In any order $L$ of $V\left(G\left(k^{\prime}, r^{\prime}\right)\right)$ which satisfies $\operatorname{wcol}_{r}\left(G\left(k^{\prime}, r^{\prime}\right)\right) \leq c$, there is some root-leaf path $P=t_{1}, \ldots, t_{m}$ such that $L\left(f\left(t_{1}\right)\right)<\ldots<L\left(f\left(t_{m}\right)\right)$.

(iii) Every bag of $T\left(k^{\prime}, r^{\prime}\right)$ contains at most $k^{\prime}+1$ vertices.

It will be convenient to define the tree decompositions first and to define the corresponding graphs as the unique graphs induced by the decomposition in the following sense. For a tree $T$ and a family of finite and non-empty sets $\left(X_{t}\right)_{t \in V(T)}$ such that if $z, s, t \in V(T)$ and $s$ is on the path of $T$ between $z$ and $t$, then $X_{z} \cap X_{t} \subseteq X_{s}$, we define the graph induced by $\left(T,\left(X_{t}\right)_{t \in V(T)}\right)$ as the graph $G$ with $V(G)=\bigcup_{t \in V(T)} X_{t}$ and $\{u, v\} \in E(G)$ if and only if $u, v \in X_{t}$ for some $t \in V(T)$. Then $\left(T,\left(X_{t}\right)_{t \in V(T)}\right)$ is a tree decomposition of $G$.

For $k^{\prime} \geq 1, r^{\prime}=1$, let $T\left(k^{\prime}, r^{\prime}\right)=: T$ be a tree of depth $k^{\prime}+1$ and branching degree $c$ with root $s$. Let $L^{T, s}$ be the natural partial tree order. Let $f: V(T) \rightarrow V$ be a bijection to some new set $V$. We define $X_{t}:=\left\{f(t): L^{T, s}\left(t^{\prime}\right) \leq L^{T, s}(t)\right\}$. Let $G\left(k^{\prime}, r^{\prime}\right)$ be the graph induced by the decomposition. The first and the third invariants clearly hold. For the second invariant, consider a simple pigeon-hole argument. For every non-leaf node $t$, the vertex $f(t)$ has $c$ neighbours $f\left(t^{\prime}\right)$ in the child bags $X_{t^{\prime}}$ of $t$. Hence some $f\left(t^{\prime}\right)$ must be larger in the order This guarantees the existence of a path as required.

For $k^{\prime}=1, r^{\prime} \geq 1$, let $T\left(k^{\prime}, r^{\prime}\right)=: T$ be a tree of depth $r^{\prime}+1$ and branching degree $c$ with root $s$ and let $f$ be as before. Let $X_{s}:=\{f(s)\}$ and for each $t^{\prime} \in V(T)$ with parent $t \in V(T)$ let $X_{t^{\prime}}:=\left\{f(t), f\left(t^{\prime}\right)\right\}$. Let $G\left(k^{\prime}, r^{\prime}\right)$ be the graph induced by the decomposition. All invariants hold by the same arguments as above. Note that $G_{1}^{1}$ is the same graph in both constructions and is hence well defined.

Now assume that $G\left(k^{\prime}, r^{\prime}-1\right)$ and $G\left(k^{\prime}-1, r^{\prime}\right)$ and their respective tree decompositions have been defined. Let $T\left(k^{\prime}, r^{\prime}\right)$ be the tree which is obtained by attaching $c$ copies of $T\left(k^{\prime}-1, r^{\prime}\right)$ as children to each leaf of $T\left(k^{\prime}, r^{\prime}-1\right)$. We define the bags that belong to the 
copy of $T\left(k^{\prime}, r^{\prime}-1\right)$, exactly as those of $T\left(k^{\prime}, r^{\prime}-1\right)$. To every bag of a copy of $T\left(k^{\prime}-1, r^{\prime}\right)$ which is attached to a leaf $z$, we add $f^{\prime}(z)$ (where $f^{\prime}$ is the bijection from $T\left(k^{\prime}, r^{\prime}-1\right)$ ). Let $G\left(k^{\prime}, r^{\prime}\right)$ be the graph induced by the decomposition.

It is easy to see how to obtain the new bijection $f$ on the whole graph such that it satisfies the invariant. It is also easy to see that each bag contains at most $k^{\prime}+1$ vertices. For invariant (ii), let $P_{1}=t_{1}, \ldots, t_{m}$ be some root-leaf path in $T\left(k^{\prime}, r^{\prime}-1\right)$ which is ordered such that $L\left(f\left(t_{1}\right)\right)<\ldots<L\left(f\left(t_{m}\right)\right)$. Let $v=f\left(t_{m}\right)$ be the unique vertex in the leaf bag in which $P_{1}$ ends. By the same argument as above, $v$ has many neighbours $s^{\prime}$ such that $f^{-1}\left(s^{\prime}\right)$ is a root of a copy of $T\left(k^{\prime}-1, r^{\prime}\right)$. One of them must be larger than $v$. In an appropriate copy we find a path $P_{2}$ with the above property by assumption. We attach the paths to find the path $P=t_{1} \ldots t_{\ell}$ in $T\left(k^{\prime}, r^{\prime}\right)$.

We finally show that WReach $r^{\prime}\left[G\left(k^{\prime}, r^{\prime}\right), L, f\left(t_{\ell}\right)\right]=\left(\begin{array}{c}r^{\prime}+k^{\prime} \\ k^{\prime}\end{array}\right)$. This is again shown by an easy induction. Using the notation of the proof of Theorem 4.2, we observe that the graph $G_{1}$ is isomorphic to $G\left(k^{\prime}, r^{\prime}-1\right)$ in $G\left(k^{\prime}, r^{\prime}\right)$ and $G_{2}$ is isomorphic to $G\left(k^{\prime}-1, r^{\prime}\right)$. Furthermore we observe that the number of vertices reached in these graphs are exactly $w\left(k^{\prime}, r^{\prime}-1\right)$ and $w\left(k^{\prime}-1, r^{\prime}\right)$, so that the upper bound is matched. Similarly one shows that $\operatorname{wcol}_{r^{\prime}}(G(k, r))=\left(\begin{array}{c}r^{\prime}+k \\ k\end{array}\right)$. The theorem follows by letting $G_{r}^{k}:=G(k, r)$.

It is proven in $[14,18]$ that for every graph $G, \operatorname{wcol}_{r}(G) \leq\left(\operatorname{col}_{r}(G)\right)^{r}$. To our knowledge, there is no example in the literature that verifies the exponential gap between $\mathrm{wcol}_{r}$ and $\mathrm{col}_{r}$. As $\operatorname{col}_{r}(G) \leq \operatorname{tw}(G)$ and $G_{r}^{k}$ contains a $k+1$-clique, Theorem 4.3 provides an example that is close to an affirmative answer for arbitrarily large generalised colouring numbers, in a rather uniform manner.

Conollary 4.4. For every $k \geq 1, r \geq 1$, there is a graph $G_{r}^{k}$ such that for all $1 \leq r^{\prime} \leq r$ we have $\operatorname{col}_{r^{\prime}}\left(G_{r}^{k}\right)=k+1$ and $\operatorname{wcol}_{r^{\prime}}\left(G_{r}^{k}\right) \geq\left(\frac{\operatorname{col}_{r^{\prime}}\left(G_{r}^{k}\right)}{r^{\prime}}\right)^{r^{\prime}}$.

Proof. Since $\operatorname{col}_{r^{\prime}}\left(G_{r}^{k}\right)=k+1$, we have

$$
\mathrm{wcol}_{r^{\prime}}\left(G_{r}^{k}\right)=\left(\begin{array}{c}
r^{\prime}+k \\
k
\end{array}\right)=\left(\begin{array}{c}
k+r^{\prime} \\
r^{\prime}
\end{array}\right) \geq\left(\frac{k+r^{\prime}}{r^{\prime}}\right)^{r^{\prime}} \geq\left(\frac{\operatorname{col}_{r^{\prime}}\left(G_{r}^{k}\right)}{r^{\prime}}\right)^{r^{\prime}} .
$$

As a further application of Theorem 4.3, we now construct a class of graphs with polynomial expansion that has super-polynomial weak colouring numbers. For a graph $G$ denote by $G^{(r)}$ the exact $r$-subdivision of $G$, that is, the graph obtained from $G$ by replacing every edge by a path of length $r+1$ (with $r$ vertices on it).

Theorem 4.5. The class $\mathcal{C}=\left\{G^{(6 \mathrm{tw}(G))}: G\right.$ graph $\}$ has polynomial expansion and super-polynomial weak colouring numbers.

Proof. Let $r \in \mathbb{N}$ and let $G$ be a graph of treewidth $t:=\operatorname{tw}(G)$. Let $H$ be the densest depth- $r$ minor of $G^{(6 t)}$. If $r \geq t$, we conclude that $\varepsilon(H) \leq t \leq r$, since $G$ as a graph of treewidth $t$ is $t$-degenerate and so are all its minors. On the other hand, if $r<t$, it is easy to see that every vertex of $H$ of degree greater than 2 is adjacent only to vertices of degree at most 2. Hence in this case $H$ is 2-degenerate. We conclude that $\nabla_{r}\left(G^{(6 t)}\right) \leq r+2$, and hence $\mathcal{C}$ is a class of polynomial (and even linear) expansion.

To prove that $\mathcal{C}$ has super-polynomial weak colouring numbers, we first relate the weak colouring numbers of the exact $k$-subdivision for any $k \in \mathbb{N}$ of a graph $G$ to the weak colouring numbers of $G$. We claim that $\operatorname{wcol}_{r \cdot(k+1)}\left(G^{(k)}\right) \geq \frac{1}{2} \mathrm{wcol}_{r}(G)$. 
To see this, consider an arbitrary order $L$ of $V\left(G^{(k)}\right)$. We construct the following order $L^{\prime}$ of $V(G)$. For $u \in V(G)$ let $u_{m} \in V\left(G^{(k)}\right)$ be the $L$-minimal vertex of $N_{k-1}(u)$ in $G^{(k)}$. For $u, v \in V(G)$ we let $L^{\prime}(u)<L^{\prime}(v)$ if $L\left(u_{m}\right)<L\left(v_{m}\right)$ or if $L\left(u_{m}\right)=L\left(v_{m}\right)$ and $L(u)<L(v)$. Now fix some $v \in V(G)$ and consider the set WReach $r\left[G, L^{\prime}, v\right]$. We observe that for each $u \in \operatorname{WReach}_{r}\left[G, L^{\prime}, v\right]$ the vertex $u_{m}$ is weakly $(r \cdot(k+1))$-reachable from $v$ in $G^{(k)}$ with respect to $L$ : we traverse the subdivided path $P$ from $v$ to $u$ in $G^{(k)}$ and, if we have not visited $u_{m}$ yet, append the path from $u$ to $u_{m}$. This path has length at most $k \cdot r+(k-1) \leq r \cdot(k+1)$ and by definition of $L^{\prime}$ and the fact that $u$ is $L^{\prime}$ minimal on the corresponding path in $G, u_{m}$ is $L$-minimal on $P$. Now when counting weakly reachable vertices, $u_{m}$ may be counted twice, once for each end of the subdivided edge it lies on. We conclude that $2 \cdot \mid$ WReach $_{r \cdot(k+1)}\left[G^{(k)}, L, v\right]|\geq|$ WReach $_{r}\left[G, L^{\prime}, v\right] \mid$ for all $v \in V(G)$. As $L$ was chosen arbitrarily, it follows that $\mathrm{wcol}_{r \cdot(k+1)}\left(G^{(k)}\right) \geq \frac{1}{2} \mathrm{wcol}_{r}(G)$.

As we have proved in Theorem 4.3 for every $k, r \in \mathbb{N}$ there exists a graph $G_{r}^{k}$ of treewidth $k$ that satisfies $\operatorname{wcol}_{r}\left(G_{r}^{k}\right)=\left(\begin{array}{c}r+k \\ r\end{array}\right)$. Now for each $t \in \mathbb{N}$ we apply the theorem to $k=r=t$, yielding a graph $H:=G_{t}^{t}$ with $\operatorname{wcol}_{t}(H)=\left(\begin{array}{c}2 t \\ t\end{array}\right)$. With our above observation we conclude that $\operatorname{wcol}_{t \cdot(6 t+1)}\left(H^{(6 t)}\right) \geq \frac{1}{2} \operatorname{wcol}_{t}(H)=\frac{1}{2}\left(\begin{array}{c}2 t \\ t\end{array}\right) \geq \frac{1}{2} \cdot \frac{4^{t}}{\sqrt{\pi t}}\left(1-\frac{1}{8 t}\right)$. Substituting $\frac{\sqrt{(1+24 r)}-1}{12}$ for $t$ gives us $\operatorname{wcol}_{r}(\mathcal{C}) \in \Omega\left((4-\varepsilon)^{\frac{\sqrt{6 r}}{6}}\right)$ for any $\varepsilon>0$, which is super-polynomial in $r$.

Theorem 4.5 answers negatively the question whether graph classes of polynomial expansion have polynomial weak colouring numbers. This question is attributed to Joret and Wood in [9]. They also ask whether graph classes of polynomial expansion have polynomial strong colouring numbers. This was proved in the meantime for graphs excluding a fixed minor [24].

5. High-Girth Regular Graphs. The goal of this section is to study the generalised colouring numbers of graph classes with constant topological expansion (such as classes excluding a topological minor). In light of ([25], Lemma 3.3), it is not surprising that such classes can have exponential weak colouring numbers. Surprisingly, we prove that, in fact, even classes of bounded degree (which are of the simplest classes that can exclude a topological minor) have superpolynomial colouring numbers, too. For this section, we let $n:=|V(G)|$.

TheOREM 5.1. Let $G$ be a d-regular graph of girth at least $4 g+1$, where $d \geq 7$. Then for every $r \leq g$,

$$
\operatorname{col}_{r}(G) \geq \frac{d}{2}\left(\frac{d-2}{4}\right)^{2^{\lfloor\log r\rfloor}-1}
$$

Proof. For an ordering $L$ of $G$, let $R_{r}(v)=\operatorname{SReach}_{r}[G, L, v] \backslash \operatorname{SReach}_{r-1}[G, L, v]$ and $U_{r}=\sum_{v \in V(G)}\left|R_{r}(v)\right|$.

Suppose that $r \leq g$ and notice that for $u, w \in R_{r}(v)$, we have that either $u \in R_{2 r}(w)$ or $w \in R_{2 r}(u)$. Therefore, every vertex $v \in V(G)$ contributes at least $\left(\begin{array}{c}\left|R_{r}(v)\right| \\ 2\end{array}\right)$ times to $U_{2 r}$. Moreover, since $r \leq g$, for every $u, w$ with $u \in R_{2 r}(w)$ there is at most one vertex $v \in V(G)$ such that $u, w \in R_{r}(v)$ (namely the middle vertex of the unique $(u, v)$-path of length $2 r$ in $G$ ). 
It follows that for every $r \leq g$,

$$
\begin{aligned}
U_{2 r} & \geq \sum_{v \in V(G)}\left(\begin{array}{c}
\left|R_{r}(v)\right| \\
2
\end{array}\right)=\frac{1}{2} \sum_{v \in V(G)}\left|R_{r}(v)\right|^{2}-\frac{1}{2} \sum_{v \in V(G)}\left|R_{r}(v)\right| \\
& \geq \frac{1}{2 n}\left(\sum_{v \in V(G)}\left|R_{r}(v)\right|\right)^{2}-\frac{1}{2} U_{r}=\frac{1}{2 n} U_{r}^{2}-\frac{1}{2} U_{r}
\end{aligned}
$$

where for the second inequality we have used the Cauchy-Schwarz inequality.

Let $c_{r}=\frac{U_{r}}{n}$. Then for every $r \leq g$, we obtain $c_{2 r} \geq \frac{1}{2} c_{r}\left(c_{r}-1\right)$. But,

$$
U_{1}=\sum_{v \in V(G)}\left|\operatorname{SReach}_{1}[G, L, v] \backslash\{v\}\right|=\frac{1}{2} d n,
$$

so that $c_{1}=\frac{d}{2}>3$, since $d \geq 7$. By induction and because $c_{2 r} \geq \frac{1}{2} c_{r}\left(c_{r}-1\right)$, for every $r=2^{r^{\prime}} \leq g$ we have $c_{2 r} \geq c_{r} \geq 3$. Therefore $c_{r} \geq c_{1}=\frac{d}{2}$. Again because $c_{2 r} \geq \frac{1}{2} c_{r}\left(c_{r}-1\right)$, for every $r=2^{r^{\prime}} \leq g$ we have

$$
c_{2 r} \geq \frac{1}{2} c_{r}^{2}-\frac{1}{2} c_{r} \geq \frac{1}{2} c_{r}^{2}-\frac{1}{d} c_{r}^{2}=\frac{d-2}{2 d} c_{r}^{2} .
$$

Then for every $r=2^{r^{\prime}} \leq g$, it easily follows by induction that $c_{r} \geq \frac{d}{2}\left(\frac{d-2}{4}\right)^{r-1}$.

Finally, let $C_{r}=\frac{1}{n} \sum_{v \in V(G)}\left|\operatorname{SReach}_{r}[G, L, v]\right|$. Then, $C_{r}=\sum_{i=1}^{r} c_{i}$. In particular, it is $C_{r} \geq c_{2\lfloor\log r\rfloor} \geq \frac{d}{2}\left(\frac{d-2}{4}\right)^{2^{\lfloor\log r\rfloor}-1}$, and hence for every $r \leq g$ there exists a vertex $v_{r} \in V(G)$ such that $\left|\operatorname{SReach}_{r}\left[G, L, v_{r}\right]\right| \geq \frac{d}{2}\left(\frac{d-2}{4}\right)^{2^{\lfloor l o g r\rfloor}-1}$. Since $L$ was arbitrary, the theorem follows. $\square$

Unfortunately, our proof above makes sense only if $d \geq 7$, which is also best possible with this approach, since for $d \leq 6$, we have $c_{1} \leq 3$. Then for the recurrence relation $c_{2 r}=\frac{1}{2} c_{r}^{2}-\frac{1}{2} c_{r}$, we get $c_{2^{i}} \leq c_{2^{i-1}}$ for every $i$ and we clearly cannot afford to have $c_{2^{i}}$ non-increasing. Somewhat better constants can be achieved if in the estimation of $c_{r}$ one uses that $c_{2^{i}} \geq c_{2^{i} 0}$, for $i \geq i_{0}>0$, instead of the relation $c_{2^{i}} \geq c_{1}$, as in our proof. Since $d \geq 7$ would be still the best that we would be able to do, we adopted the simpler approach for easier readability.

Actually, by combining a known result for the $\nabla_{r}$ of high-girth regular graphs ([4],[18] Exercise 4.2) and ([25], Lemma 3.3), we get exponential lower bounds for the weak colouring number of high-girth $d$-regular graphs, already for $d \geq 3$. In particular, for a 3 -regular graph $G$ of high enough girth, $\operatorname{wcol}_{r}(G) \geq 3 \cdot 2^{\lfloor r / 4\rfloor-1}$. The methods above can be extended to get appropriate bounds in terms of their degree for regular graphs of higher degree, but by adopting a more straightforward approach, we get better bounds for high-girth $d$-regular graphs for $d \geq 4$.

THEOREM 5.2. Let $G$ be a d-regular graph of girth at least $2 g+1$, where $d \geq 4$. Then for every $r \leq g$,

$$
\operatorname{wcol}_{r}(G) \geq \frac{d}{d-3}\left(\left(\frac{d-1}{2}\right)^{r}-1\right) .
$$


Proof. Let $L$ be an ordering of $G$. For $u, v \in V(G)$ with $d(u, v) \leq r$, let $P_{u v}$ be the unique $(u, v)$-path of length at most $r$, due to the girth of $G$. Let

$$
Q_{r}(v)=\text { WReach }_{r}[G, L, v] \backslash \text { WReach }_{r-1}[G, L, v],
$$

and define $S_{r}=\sum_{v \in V(G)}\left|Q_{r}(v)\right|$. For $r \leq g-1$, a vertex $u \in Q_{r}(v)$ and $w \in N(v) \backslash V\left(P_{u v}\right)$, it holds that either $w \in Q_{r+1}(u)$ or $u \in Q_{r+1}(w)$. Notice that $\left|N(v) \backslash V\left(P_{u v}\right)\right|=d-1$ and that $P_{v u}$ and $P_{u w}$ are unique. Therefore, every pair of vertices $v, u$ with $u \in Q_{r}(v)$ corresponds to at least $d-1$ pairs of vertices $u, w$ with either $u \in Q_{r+1}(w)$ or $w \in Q_{r+1}(u)$ and hence contributes at least $d-1$ times to $S_{r+1}$. Since every path of length $r+1$ contains exactly two subpaths of length $r$, we have for every $r \leq g-1$ that

$$
2 S_{r+1} \geq(d-1) S_{r}
$$

Let $w_{r}=\frac{S_{r}}{n}$. Then, for every $r \leq g-1$ we have $w_{r+1} \geq \frac{d-1}{2} w_{r}$.

But,

$$
\sum_{v \in V(G)} \mid \text { WReach }_{1}[G, L, v] \backslash\{v\} \mid=\frac{1}{2} d n,
$$

so that $w_{1}=\frac{d}{2}$.

It easily follows by induction that for every $r \leq g$, we have $w_{r} \geq \frac{d}{2}\left(\frac{d-1}{2}\right)^{r-1}$. Finally, let $W_{r}=\frac{1}{n} \sum_{v \in V(G)}\left|\operatorname{WReach}_{r}[G, L, v]\right|$. Then,

$$
W_{r}=\sum_{i=1}^{r} w_{i} \geq \sum_{i=1}^{r} \frac{d}{2}\left(\frac{d-1}{2}\right)^{i-1}=\frac{d}{d-3}\left(\left(\frac{d-1}{2}\right)^{r}-1\right),
$$

and hence we have that for every $r \leq g$ there exists a vertex $v_{r} \in V(G)$ such that $\left|\mathrm{WReach}_{r}\left[G, L, v_{r}\right]\right| \geq \frac{d}{d-3}\left(\left(\frac{d-1}{2}\right)^{r}-1\right)$. Since $L$ was arbitrary, the theorem follows.

REMARK 5.3. Notice that for every d-regular graph $G$ and every radius $r$, we have $\operatorname{adm}_{r}(G) \leq \Delta(G)+1=d+1$, so by Theorem 5.1 for every $d \geq 7$ and every $r \leq g$, the $d$-regular graphs of girth at least $2 g+1$ verify the exponential gap between adm $m_{r}, \Delta(G)$ and $\mathrm{Col}_{r}, \mathrm{wcol}_{r}$ of the known relations from Section 2.

6. Neighbourhood Covers. Neighbourhood covers of small radius and small size play a key role in the design of many data structures for distributed systems. For references about neighbourhood covers, we refer the reader to [1].

For $r \in \mathbb{N}$, an $r$-neighbourhood cover $\mathcal{X}$ of a graph $G$ is a set of connected subgraphs of $G$ called clusters, such that for every vertex $v \in V(G)$ there is some $X \in \mathcal{X}$ with $N_{r}(v) \subseteq X$.

The radius $\operatorname{rad}(\mathcal{X})$ of a cover $\mathcal{X}$ is the maximum radius of any of its clusters. The degree $d^{\mathcal{X}}(v)$ of $v$ in $\mathcal{X}$ is the number of clusters that contain $v$. A class $\mathcal{C}$ admits sparse neighbourhood covers if for every $r \in \mathbb{N}$, there exists $c \in \mathbb{N}$ such that for all $\varepsilon>0$, there is $n_{0} \in \mathbb{N}$ such that for all $G \in \mathcal{C}$ of order at least $n_{0}$, there exists an $r$-neighbourhood cover of radius at most $c \cdot r$ and degree at most $|V(G)|^{\varepsilon}$. For any graph $G$, one can construct an $r$-neighbourhood cover of radius $2 r-1$ and degree $2 k \cdot|V(G)|^{1 / r}$ and asymptotically these bounds cannot be improved [23].

Theorem 6.1 (Theorem 16.2.4 of [21], [23]). For every $r$ and $k \geq 3$, there exist infinitely many graphs $G$ for which every $r$-neighbourhood cover of radius at most $k$ has degree $\Omega\left(|V(G)|^{1 / k}\right)$. 
For restricted classes of graphs, better covers exist. The most general results are that a class excluding a complete graph on $t$ vertices as a minor admits an $r$-neighbourhood cover of radius $\mathcal{O}\left(t^{2} \cdot r\right)$ and degree $2^{\mathcal{O}(t)} t$ ! [1], as well as the following result from [12].

THEOREM 6.2 ([12]). Let $\mathcal{C}$ be a nowhere dense class of graphs. There is a function $f$ such that for all $r \in \mathbb{N}$ and $\varepsilon>0$ and all graphs $G \in \mathcal{C}$ with $|V(G)| \geq f(r, \varepsilon)$, there exists an $r$-neighbourhood cover of radius at most $2 r$ and maximum degree at most $|V(G)|^{\varepsilon}$. More precisely, if $\mathrm{wcol}_{2 r}(G)=d$, then there exists an $r$-neighbourhood cover of radius at most $2 r$ and maximum degree at most $d$.

Hence our new bounds for the weak colouring numbers on restricted graph classes immediately imply improved $r$-neighbourhood covers on these classes.

We show that for monotone classes the converse of Theorem 6.2 is also true. We first observe that the lower bounds in Theorem 6.1 come from a well known somewhere dense class.

LEMMA 6.3. Let $d \geq 1, k \geq 2$ and let $G$ be a graph of girth at least $k+1$ and edge density at least $d$. Then every 1-neighbourhood cover of radius at most $k$ has degree at least $d$.

LEMMA 6.4 ([17]). Let $r \geq 5$. There are infinitely many graphs $G$ of girth at least $4 r$ with edge density at least $c_{0} \cdot|V(G)|^{1 /(3(r-1))}$ for some constant $c_{0}>0$.

THEOREM 6.5. If $\mathcal{C}$ is somewhere dense and monotone, then $\mathcal{C}$ does not admit sparse neighbourhood covers.

Proof. Let $\mathcal{C}$ be somewhere dense. Then for some integer $s$, all graphs $H$ are topological depth- $s$ minors of a graph $G \in \mathcal{C}$. Assume towards a contradiction that $\mathcal{C}$ admits a sparse neighbourhood cover. Then for every $G \in \mathcal{C}$ there is an $r \cdot s$-neighbourhood cover of radius $c \cdot r \cdot s$ (for some constant $c$ ) which for every $\varepsilon>0$ has degree at most $|V(G)|^{\varepsilon}$ if $G$ is sufficiently large. Fix some $r \geq 5$.

Claim 1. If an $s$-subdivision of $H$ admits an $r \cdot s$-neighbourhood cover of radius $c \cdot r \cdot s$ and degree $d$, then $H$ admits an $r$-neighbourhood cover of radius $c \cdot r \cdot s$ and degree $d$.

Proof. Let $G$ be an $s$-subdivision of $H$ and let $\mathcal{X}$ be an $r \cdot s$-neighbourhood cover of $G$. Let $\mathcal{Y}$ be the projected cover which for every $X \in \mathcal{X}$ has a cluster $Y(X):=X \cap V(H)$

Then $\mathcal{Y}$ is an $r$-neighbourhood cover of radius $c \cdot r \cdot s$ and degree $d$ : Clearly, every $Y(X)$ is connected and has radius at most $c \cdot r \cdot s$. Let $v \in V(G)$. There is a cluster $X \in \mathcal{X}$ such that $N_{r s}^{G}(v) \subseteq X$. Then $N_{r}^{H}(v)=N_{r s}^{G}(v) \cap V(H) \subseteq X \cap V(H)=Y(X)$. Finally, the degree of $\mathcal{Y}$ is at most $d$, as every vertex $v$ of $H$ is exactly in those clusters $Y(X)$ with $v \in X$.

Let $H$ be a large graph of girth greater than $c \cdot r \cdot s$ with edge density $d=c_{0} \cdot|V(H)|^{1 /(\text { crs })}$ for some constant $c_{0}$. Such $H$ exists by Lemma 6.4 and $H$ does not admit an $r \cdot s$-neighbourhood cover of radius $c \cdot r \cdot s$ and degree $d$ by Lemma 6.3. As $\mathcal{C}$ is monotone, an $s$-subdivision of $H$ is a graph $G \in \mathcal{C}$ with $|V(G)| \leq|V(H)|+s \cdot|E(H)| \leq 2 c_{0} s|V(H)|^{1+1 /(c r s)}$.

By assumption, $G$ admits an $r \cdot s$-neighbourhood cover of radius at most $c \cdot r \cdot s$ and degree at most $|V(G)|^{\varepsilon}$ for $\varepsilon=1 /(2 c r s)$ if $G$ is large enough. It follows from Claim 1 that $H$ has a cover of radius $c \cdot r \cdot s$ and degree at most

$$
\begin{aligned}
|V(G)|^{\varepsilon} \leq\left(2 c_{0} s|V(H)|^{1+1 /(c r s)}\right)^{\varepsilon} & =\left(2 c_{0} s\right)^{\varepsilon} \cdot|V(H)|^{\varepsilon+\varepsilon /(c r s)} \\
& <c_{0}|V(H)|^{2 \varepsilon}=c_{0}|V(H)|^{1 /(c r s)}
\end{aligned}
$$

for sufficiently large $H$. A contradiction. 
A similiar characterisation for classes of bounded expansion was found by Nešetřil and Ossona de Mendez [19].

7. The Complexity of Computing $\mathbf{w c o l}_{\mathbf{r}}(\mathbf{G})$. Unlike computing the degeneracy of a graph $G$, i.e. $\operatorname{wcol}_{1}(G)+1$, deciding whether $\operatorname{wcol}_{r}(G)=k$ turns out to be NP-complete for all $r \geq 3$. The case $r=2$ remains an open question. Clearly, the problem is in NP, hence it remains to show NP-hardness. The proof is a straightforward modification of a proof of Pothen [22], showing that computing a minimum elimination tree height problem is NPcomplete. It is based on a reduction from the NP-complete problem BALANCED COMPLETE BIPARTITE SUBGRAPH (BCBS, problem GT24 of [10]): given a bipartite graph $G$ and a positive integer $k$, decide whether there are two disjoint subsets $W_{1}, W_{2} \subseteq V(G)$ such that $\left|W_{1}\right|=\left|W_{2}\right|=k$ and such that $u \in W_{1}, v \in W_{2}$ implies $\{u, v\} \in E(G)$. For a graph $G$, let $\bar{G}$ be its complement graph.

Lemma 7.1. Let $G=\left(V_{1} \cup V_{2}, E\right)$ be a bipartite $n$-vertex graph and let $k \in \mathbb{N}$. Then $G$ has a balanced complete bipartite subgraph with partitions $W_{1}, W_{2}$ of size $k$ if and only if $\operatorname{wcol}_{r}(\bar{G})=\operatorname{wcol}_{3}(\bar{G}) \leq n-k$ for all $r \geq 3$.

Proof. $\bar{G}$ is the complement of a bipartite graph, i.e. $V_{1}$ and $V_{2}$ induce complete subgraphs in $\bar{G}$ and there are possibly further edges between vertices of $V_{1}$ and $V_{2}$. Thus, for any two vertices $u, v$ which are connected in $\bar{G}$ by a path $P$, there is a subpath of $P$ between $u$ and $v$ of length at most 3 . Hence $\operatorname{wcol}_{r}(\bar{G})=\operatorname{wcol}_{3}(\bar{G})$ for any $r \geq 3$ and it suffices to show that $G$ has a balanced complete bipartite subgraph with partitions $W_{1}, W_{2}$ of size $k$ if and only $\operatorname{wcol}_{3}(\bar{G})=n-k$.

First assume that there are sets $W_{1} \subseteq V_{1}, W_{2} \subseteq V_{2}$ with $\left|W_{1}\right|=\left|W_{2}\right|=k$ and such that for all $u \in W_{1}, v \in W_{2}$ there is an edge $\{u, v\} \in E(G)$. Let $L$ be some order which satisfies $L(u)<L(v)$ if $u \in V(\bar{G}) \backslash\left(W_{1} \cup W_{2}\right)$ and $v \in W_{1} \cup W_{2}$ and $L(v)<L(w)$ if $v \in W_{1}$ and $w \in W_{2}$. Then any vertex from $V(\bar{G}) \backslash\left(W_{1} \cup W_{2}\right)$ weakly reaches at most $n-2 k$ vertices and any vertex from $W_{i}$ for $1 \leq i \leq 2$ weakly reaches at most $n-k$ vertices.

Now let $L$ be an order with WReach ${ }_{3}[\bar{G}, L, v] \leq n-k$ for all $v \in V(G)$. Assume without loss of generality that $V(G)=\left\{v_{1}, v_{2}, \ldots, v_{n}\right\}$ with $L\left(v_{i}\right)<L\left(v_{i+1}\right)$ for all $i<n$. Denote by $\bar{G}_{i}$ the subgraph $\bar{G}\left[\left\{v_{i}, \ldots, v_{n}\right\}\right]$ and let $V_{1}^{i}:=V\left(\bar{G}_{i}\right) \cap V_{1}$ and $V_{2}^{i}:=V\left(\bar{G}_{i}\right) \cap V_{2}$. Let $\ell \geq 1$ be minimal such that there is no edge between $V_{1}^{\ell}$ and $V_{2}^{\ell}$ in $\bar{G}$. It exists because one of $V_{1}^{n}$ or $V_{2}^{n}$ is empty. Clearly, $V_{1}^{\ell}$ and $V_{2}^{\ell}$ induce a complete bipartite graph in $G$. Let $j_{1}:=\left|V_{1}^{\ell}\right|$ and $j_{2}:=\left|V_{2}^{\ell}\right|$. We show that $j_{1}, j_{2} \geq k$. It is easy to see that $\operatorname{WReach}_{3}\left[\bar{G}, L, w_{1}\right] \leq \ell+j_{1}$ for the maximal element $w_{1} \in V_{1}^{\ell}$ and WReach $3\left[\bar{G}, L, w_{2}\right] \leq \ell+j_{2}$ for the maximal element $w_{2} \in V_{2}^{\ell}$. We have $j_{1}+j_{2}=n-\ell$ and, without loss of generality, $\ell+j_{1} \leq \ell+j_{2} \leq n-k$. Hence $j_{1} \leq j_{2} \leq n-\ell-k=j_{1}+j_{2}-k$, which implies both $j_{1} \geq k$ and $j_{2} \geq k$.

The above reduction is polynomial time computable, so we obtain the following theorem.

TheOREM 7.2. Given a graph $G$ and $k, r \in \mathbb{N}, r \geq 3$, it is NP-complete to decide whether $\operatorname{wcol}_{r}(G)=k$.

8. Concluding Remarks. We have studied generalised colouring numbers of graphs and proved new upper and lower bounds. These colouring numbers can be used to characterise nowhere dense graph classes, and they are directly related to bounds on the degrees of neighbourhood covers for such classes. 
For graph classes of bounded expansion, the colouring numbers do not depend on the size of the input graphs, and we may view them as functions of $r$. For classes of treewidth at most $k$ we have obtained a polynomial bound, roughly $r^{k}$, for the weak colouring number, whereas for classes that exclude a fixed graph as a topological minor we have an exponential bound $\operatorname{wcol}_{r}(G) \in \mathcal{O}\left(\left(c_{\mathcal{C}} \cdot r\right)^{r}\right)$, where $c_{\mathcal{C}}$ is a constant depending only on the class $\mathcal{C}$. Our lower bound for bounded degree classes shows that we cannot hope to improve the latter bound substantially. Finally, we negatively answered a question of Joret and Wood, showing that there exist classes of polynomial expansion which have super-polynomial weak colouring numbers.

Acknowledgements. We thank Michał Pilipczuk and Felix Reidl for useful discussions on graph classes of polynomial expansion.

\section{REFERENCES}

[1] I. Abraham, C. Gavoille, D. Malkhi, and U. Wieder, Strong-diameter decompositions of minor free graphs, in Proceedings of the nineteenth annual ACM symposium on Parallel algorithms and architectures, ACM, 2007, pp. 16-24.

[2] A. Dawar and S. Kreutzer, Domination problems in nowhere-dense graph classes, in Proceedings of the 29th Conference on Foundations of Software Technology and Theoretical Computer Science, vol. 4 of LIPIcs, Schloss Dagstuhl - Leibniz-Zentrum für Informatik, 2009, pp. 157-168.

[3] R. Diestel, Graph Theory, 4th Edition, vol. 173 of Graduate texts in mathematics, Springer, 2012.

[4] R. Diestel and C. Rempel, Dense minors in graphs of large girth., Combinatorica, 25 (2004), pp. 111116.

[5] Z. Dvoř́́k, Asymptotical structure of combinatorial objects, PhD thesis, Charles University, Prague, 2007.

[6] Z. DVořÁK, Constant-factor approximation of the domination number in sparse graphs, European Journal of Combinatorics, 34 (2013), pp. 833-840.

[7] Z. Dvořák, D. KRÁL, And R. Thomas, Testing first-order properties for subclasses of sparse graphs, Journal of the ACM, 60 (2013), pp. 36:1-36:24.

[8] K. Eickmeyer, A. C. Giannopoulou, S. Kreutzer, O. Kwon, M. Pilipczuk, R. Rabinovich, and S. SieBertZ, Neighborhood complexity and kernelization for nowhere dense classes of graphs, in 44th International Colloquium on Automata, Languages, and Programming, ICALP, 2017, pp. 63:1-63:14

[9] L. Esperet And J. Raymond, Polynomial expansion and sublinear separators, European Journal of Combinatorics, 69 (2018), pp. 49-53.

[10] M. R. Garey And D. S. Johnson, Computers and Intractability: A Guide to the Theory of NPCompleteness, W. H. Freeman, 1979.

[11] M. Grohe, S. Kreutzer, And S. Siebertz, Characterisations of nowhere dense graphs, in Proceedings of the 32nd IARCS Annual Conference on Foundations of Software Technology and Theoretical Computer Science, vol. 24 of LIPIcs, Schloss Dagstuhl - Leibniz-Zentrum für Informatik, 2013, pp. 21-40.

[12] M. Grohe, S. Kreutzer, And S. Siebertz, Deciding first-order properties of nowhere dense graphs, Journal of the ACM, 64 (2017), pp. 17:1-17:32.

[13] H. A. Kierstead AND W. T. TROTTER, Planar graph coloring with an uncooperative partner, Journal of Graph Theory, 18 (1994), pp. 569-584.

[14] H. A. Kierstead AND D. YANG, Orderings on graphs and game coloring number, Order, 20 (2003), pp. $255-264$.

[15] S. Kreutzer, Algorithmic meta-theorems, in Finite and Algorithmic Model Theory, London Mathematical Society Lecture Note Series, Cambridge University Press, 2011, ch. 5, pp. 177-270.

[16] S. Kreutzer, R. Rabinovich, And S. Siebertz, Polynomial kernels and wideness properties of nowhere dense graph classes, in Proceedings of the Twenty-Eighth Annual ACM-SIAM Symposium on Discrete Algorithms, SODA, 2017, pp. 1533-1545.

[17] F. Lazebnik, V. A. Ustimenko, and A. J. Woldar, A new series of dense graphs of high girth, Bulletin of the American Mathematical Society, 32 (1995), pp. 73-79.

[18] J. NeŠEtřil And P. Ossona de Mendez, Sparsity, Springer, 2012. 
[19] J. NešEtřil And P. Ossona de Mendez, On low tree-depth decompositions, Graphs and Combinatorics, 31 (2015), pp. 1941-1963.

[20] J. NeŠEtřil and P. Ossona de Mendez, On nowhere dense graphs, European Journal of Combinatorics, 32 (2011), pp. 600-617.

[21] D. Peleg, Distributed computing, SIAM Monographs on discrete mathematics and applications, 5 (2000).

[22] A. Pothen, The complexity of optimal elimination trees, Pennsylvania State University, Department of Computer Science, 1988.

[23] M. Thorup And U. Zwick, Approximate distance oracles, Journal of the ACM, 52 (2005), pp. 1-24.

[24] J. van den Heuvel, P. Ossona de Mendez, D. A. Quiroz, R. Rabinovich, and S. Siebertz, On the generalised colouring numbers of graphs that exclude a fixed minor, European Journal of Combinatorics, 66 (2017), pp. 129-144.

[25] X. ZHU, Colouring graphs with bounded generalized colouring number, Discrete Mathematics, 309 (2009), pp. $5562-5568$. 